\title{
Binaural Unmasking of Multi-channel Stimuli in Bilateral Cochlear Implant Users
}

\author{
Lieselot Van Deun $^{1}$, Astrid van Wieringen ${ }^{1}$, Tom Francart ${ }^{1}$, Andreas Büchner $^{2}$, Thomas Lenarz ${ }^{2}$, \\ AND JAN WOUTERs ${ }^{1}$ \\ ${ }^{1}$ ExpORL/Department of Neurosciences, K.U.Leuven, OENN2, Herestraat 49 bus 721, 3000 Leuven, Belgium \\ ${ }^{2}$ Department of Otolaryngology, Medical University of Hannover, Carl-Neuberg-Strasse 1, 30625 Hannover, Germany
}

Received: 8 November 2010; Accepted: 24 May 2011; Online publication: 9 June 2011

\begin{abstract}
Previous work suggests that bilateral cochlear implant users are sensitive to interaural cues if experimental speech processors are used to preserve accurate interaural information in the electrical stimulation pattern. Binaural unmasking occurs in adults and children when an interaural delay is applied to the envelope of a high-rate pulse train. Nevertheless, for speech perception, binaural unmasking benefits have not been demonstrated consistently, even with coordinated stimulation at both ears. The present study aimed at bridging the gap between basic psychophysical performance on binaural signal detection tasks on the one hand and binaural perception of speech in noise on the other hand. Therefore, binaural signal detection was expanded to multi-channel stimulation and biologically relevant interaural delays. A harmonic complex, consisting of three sinusoids $(125,250$, and $375 \mathrm{~Hz})$, was added to three 125-Hz-wide noise bands centered on the sinusoids. When an interaural delay of $700 \mu$ s was introduced, an average BMLD of $3 \mathrm{~dB}$ was established. Outcomes are promising in view of real-life benefits. Future research should investigate the generalization of the observed benefits for signal detection to speech perception in everyday listening situations and determine the importance of coordination of bilateral speech processors and accentuation of envelope cues.
\end{abstract}

Sources of support: K.U.Leuven Research Council and IWT Vlaanderen

Correspondence to: Lieselot Van Deun - ExpORL/Department of Neurosciences - K.U.Leuven - O\&N2, Herestraat 49 bus 721, 3000 Leuven, Belgium. Telephone: +32-16-330495; fax: +32-16-330486; email: lieselot.vandeun@med.kuleuven.be
Keywords: binaural, masking level difference, electrical stimulation

\section{INTRODUCTION}

The benefits of bilateral cochlear implants (CIs) have been the subject of numerous recent investigations. Particularly, the contribution of true binaural processes - in which information from both ears is combined in the brain-to speech perception is questioned. Normal-hearing listeners benefit from spatial separation of a target speech source and disturbing noise sources. This benefit results from an improvement in signal-to-noise ratio at one ear (better-ear effect) and from noise suppression that can occur when speech and noise have different interaural phase and level relationships (binaural unmasking). In CI users, most bilateral benefits seem to arise from better-ear effects, whereas limited true binaural hearing abilities have been established with clinical speech processors (e.g., Buss et al. 2008; Laszig et al. 2004; Litovsky et al. 2006; Schleich et al. 2004; Van Deun et al. 2010b). It is unknown how much of these limitations originate from technical aspects, related to the independent functioning of left and right speech processors. With coordinated stimulation of a single electrode pair, binaural unmasking has been demonstrated (Long et al. 2006; Lu et al. 2010; Van Deun et al. 2009). In those studies, binaural masking level differences (BMLDs) were measured as the difference in detection threshold for pulse trains with sinusoidal modulations that are in phase at the two ears (diotic) versus out of phase (dichotic), in the 
presence of diotic noise. Unmasking effects of 5 to $10 \mathrm{~dB}$ have been established in adults (Long et al. 2006) and children (Van Deun et al. 2009), for different frequencies, and for different places along the cochlea (Lu et al. 2010). However, van Hoesel et al. (2008) did not find a binaural intelligibility level difference (BILD) for speech signals with coordinated stimulation, even when temporal fine structure cues were provided to the CI listeners. Several factors could possibly account for the discrepancies between the BMLD and the BILD studies. First, signal detection and speech understanding are tasks tapping into different processes and may rely on different auditory cues. Second, speech has a variable and complex temporal and spectral structure, whereas BMLDs were measured with basic stimuli. The speech and noise stimuli that were used in the BILD study (van Hoesel et al. 2008) comprised fast fluctuations and activated multiple channels in the cochlear implant. BMLDs, to the contrary, were measured with lowfrequency signals and narrow bands of noise and have never been measured with electrical stimulation on multiple channels. Third, relatively large interaural delays - on the order of milliseconds - have been used in the BMLD studies, as compared to natural head-width delays - up to $700 \mu \mathrm{s}$ - which were used in the BILD study of van Hoesel et al. (2008).

In this study, steps were taken to bridge the gap between the single-channel, narrowband BMLDs and the broadband BILDs by measuring BMLDs for broadband stimuli presented to multiple electrodes in a controlled setup. Three electrode pairs were stimulated with a harmonic complex consisting of three sinusoids. Stimulus characteristics were chosen to be similar to those of speech signals (vowels). The interaural time difference (ITD) was gradually reduced to further approach realistic situations. It was hypothesized that a BMLD would be measured with controlled electrical stimulation of multiple channels, but that the size of the BMLD would be reduced significantly as a result of the larger bandwidth, shorter interaural delay, and stimulation of multiple channels. Particularly, spread of excitation and electrode interaction might disturb the stimulation pattern within channels (McKay and McDermott 1996) and affect the salience of the interaural cues. This study will provide insight into the opportunities to induce binaural sensitivity to complex stimuli that are more representative of everyday signals.

\section{MATERIALS AND METHODS}

\section{Subjects}

Seven cochlear implant patients, three young adolescents (10-17 year) and four adults, participated in this study. All subjects were daily users of bilateral Nucleus cochlear implants. Details on their hearing history and speech processors are given in Table 1. The children participated in previous research on sound localization (Van Deun et al. 2010a) and single-channel binaural masking level differences (Van Deun et al. 2009) and their IDs are similar to the ones used in those studies. Children were tested at their home; adults were tested at the hearing center of the Medical University of Hannover, Germany. Testing was approved by the K.U. Leuven Medical Ethical Committee and was in accordance with the Declaration of Helsinki (1975).

\section{Setup}

The test setup consisted of two experimental CI speech processors (L34) controlled using the NIC interface, provided by Cochlear Ltd. The processors were synchronized to each other (resolution $<200 \mathrm{~ns}$ ) and connected to a laptop. Version 3 of the APEX program, developed at ExpORL-K.U.Leuven (Francart et al. 2008), was used for test management.

\section{Stimuli}

Stimuli were created in Matlab and checked on the oscilloscope (Le Croy 9304AM). Fixed-rate electrical pulse trains were modulated with the combination of a sine wave and a noise band. The sine wave and masking noise were generated digitally at a sampling frequency of $44.1 \mathrm{kHz}$. In the $\mathrm{N}_{0} \mathrm{~S}_{0}$ condition, the processing scheme was identical for the left and right ears, with an identical or diotic sinusoid $\left(\mathrm{S}_{0}\right)$ and noise $\left(\mathrm{N}_{0}\right)$ at both ears. In the $\mathrm{N}_{0} \mathrm{~S}_{\tau}$ condition, the sinusoid was delayed in the right channel $\left(\mathrm{S}_{\tau}\right)$, before being added to the diotic noise $\left(\mathrm{N}_{0}\right)$. The first line of the block diagram of Figure 1 shows the conversion of the digitally sampled waveforms (corresponding to acoustic signals) into electrical pulses for one ear and one stimulation channel. Processing was similar to the CIS processing strategy (Wilson et al. 1991) on which most current clinical speech processing strategies are based. The combined signal (sinusoid plus noise) was half-wave rectified - to mimic the behavior of auditory nerve fibers, which fire only to the positive phase of low-frequency sounds-and low-pass filtered at $500 \mathrm{~Hz}$ with a fourth-order Butterworth filter. Subsequently, the top $30 \mathrm{~dB}$ of the signal was selected to be mapped into the dynamic range of the subject, applying a compression similar to that used in clinical systems, according to Formula 1.

$$
y=1-\exp (-5.0 \times x)
$$

with $x$ and $y$ the amplitude of the input and output signal, respectively. 
TABLE 1

\begin{tabular}{|c|c|c|c|c|c|c|c|c|c|c|c|c|c|}
\hline \multicolumn{14}{|c|}{ Subject details } \\
\hline$I D$ & $\begin{array}{l}\text { Age } \\
(y r)\end{array}$ & $\begin{array}{l}\text { Age at } \\
O O D \\
(y r, m o)\end{array}$ & Etiology & $\begin{array}{l}\text { Age at } \mathrm{Cl} \\
\text { switch } \\
\text { on - right } \\
(y r, m o)\end{array}$ & $\begin{array}{l}\text { Age at } \mathrm{Cl} \\
\text { switch } \\
\text { on-left } \\
(y r, m o)\end{array}$ & $\begin{array}{l}\text { Delay } \\
\text { between } \\
\text { Cls }(y r, m o)\end{array}$ & $\begin{array}{l}\text { Duration use } \\
\mathrm{Cl} 1(\mathrm{yr})\end{array}$ & $\begin{array}{l}\text { Duration use } \\
\mathrm{Cl} 2(\mathrm{yr})\end{array}$ & $\begin{array}{l}\text { Speech } \\
\text { proc-right }\end{array}$ & $\begin{array}{l}\text { Speech } \\
\text { proc-left }\end{array}$ & $\begin{array}{l}\text { Speech } \\
\text { proc } \\
\text { strategy }\end{array}$ & $\begin{array}{l}\text { HA use } \\
\text { before } C l \\
1 \text { (yr,mo) }\end{array}$ & $\begin{array}{l}\text { HA use } \\
\text { before } \mathrm{Cl} 2 \\
(y r, m o)\end{array}$ \\
\hline $\mathrm{Cl} 3$ & 10 & 0,0 & CMV & 2,0 & 4,4 & 2,4 & 8 & 6 & $\mathrm{Fr}$ & $\mathrm{Fr}$ & ACE & 1,7 & 3,11 \\
\hline $\mathrm{Cl} 5$ & 11 & 0,7 & Men & 1,8 & 5,5 & 3,9 & 9 & 6 & $\mathrm{Fr}$ & $\mathrm{Fr}$ & ACE & 0,0 & 0,0 \\
\hline $\mathrm{Cl} 7$ & 17 & 0,0 & LVAS & 5,11 & 11,1 & 5,2 & 11 & 6 & $\mathrm{Fr}$ & $\mathrm{Fr}$ & ACE & 4,11 & 10,1 \\
\hline Cla1 & 43 & $27,9 / 39,9$ & Genetic & 38,8 & 42,0 & 3,4 & 4 & 1 & $\mathrm{Fr}$ & $\mathrm{Fr}$ & ACE & 30,10 & 34,2 \\
\hline Cla2 & 62 & 52,3 & Idiopathic & 57,2 & 60,1 & 2,11 & 5 & 2 & $\mathrm{Fr}$ & $\mathrm{Fr}$ & ACE & 21,11 & 24,10 \\
\hline Cla3 & 57 & 47,7 & Trauma & 48,0 & 47,11 & 0,1 & 9 & 9 & $3 G$ & $3 G$ & ACE & 0,0 & 0,0 \\
\hline Cla4 & 46 & 35,9 & Infection & 43,8 & 45,10 & 2,2 & 2 & 0,4 & $\mathrm{Fr}$ & CP810 & ACE & 17,11 & 20,1 \\
\hline
\end{tabular}

yr years, mo months, $O O D$ onset of deafness, $\mathrm{Cl}$ cochlear implant, $\mathrm{HA}$ hearing aid, Speech proc speech processor, Fr Nucleus freedom, $3 G$ Nucleus Esprit3G, CP810 Nucleus CP810, ACE advanced combination encoder, CMV cytomegalovirus, Men meningitis, LVAS large vestibular aqueduct syndrome (progressive)

An electrical pulse train of 1,000 pps was then modulated with this signal. The resampling from 44.1 to $1 \mathrm{kHz}$ was done by taking the sample values closest in time to the target periodicity of $1 \mathrm{~ms}$ for the $1-\mathrm{kHz}$ rate (identical to the procedure used in Long et al. (2006)). Pulses were biphasic, with a phase width of $25 \mu \mathrm{s}$ and an inter-phase gap of $8 \mu \mathrm{s}$, and were presented in monopolar mode $(\mathrm{MP} 1+2)$. Pulses were presented to one or three electrodes at each ear.

Single-channel BMLDs were first measured with the same stimulus characteristics as in previous research (Van Deun et al. 2009), but in an adaptive instead of constant-level procedure. Subsequently, the interaural delay was reduced and multi-channel stimulation was applied. The specific characteristics of the different stimuli were as follows:

a. Single-channel stimuli: a $125-\mathrm{Hz}$ sinusoid was embedded in a narrowband masker, $50 \mathrm{~Hz}$ wide and centered on $125 \mathrm{~Hz}$. The noise masker was created by band-pass filtering white noise with a fourthorder Butterworth filter with cutoff frequencies of 100 and $150 \mathrm{~Hz}$. The sinusoid and noise were ramped on and off over $50 \mathrm{~ms}$ with a cosine window and had a duration of 300 and $400 \mathrm{~ms}$, respectively. The 1,000-pps pulse train, modulated with the combined and processed signal and noise, was presented to one electrode in the middle of the electrode array, electrode 13 (starting to count from the most basal electrode), both on the left and right sides. In the diotic condition, the same signal and noise were presented to each ear. In the dichotic conditions, the sinusoid was delayed with either 4,2 , or $0.7 \mathrm{~ms}$ at the right ear. Figure 2 shows the enlarged version of the pulses applied to one left and one right electrode for the 4-ms-delayed dichotic $\left(\mathrm{N}_{0} \mathrm{~S}_{\tau}\right)$ stimulus (signal plus noise) for different signal-to-noise ratios (SNRs), after compression. Note that all SNR levels in this manuscript refer to the levels in $\mathrm{dB}$ for the "acoustic" input signals, which are converted into current units according to the dynamic range of the listener.

b. Multi-channel stimuli: the stimulus at each electrode was similar to the single-channel stimuli. Each of three adjacent electrodes was stimulated with a 1,000 -pps pulse train, modulated with a sinusoid in a noise band. The sinusoids were the first three harmonics of a complex signal with a fundamental frequency of $125 \mathrm{~Hz}$. This means that a sinusoid of $125 \mathrm{~Hz}$ was presented to the most apical (14) of the three electrodes, a sinusoid of $250 \mathrm{~Hz}$ to the middle electrode (13), and a sinusoid of $375 \mathrm{~Hz}$ to the most basal electrode (12). In each channel, a 125-Hz-wide noise band, centered on the sinusoid frequency in that channel, was added. Noise cutoff frequencies were therefore 62.5 and $187.5 \mathrm{~Hz}$,

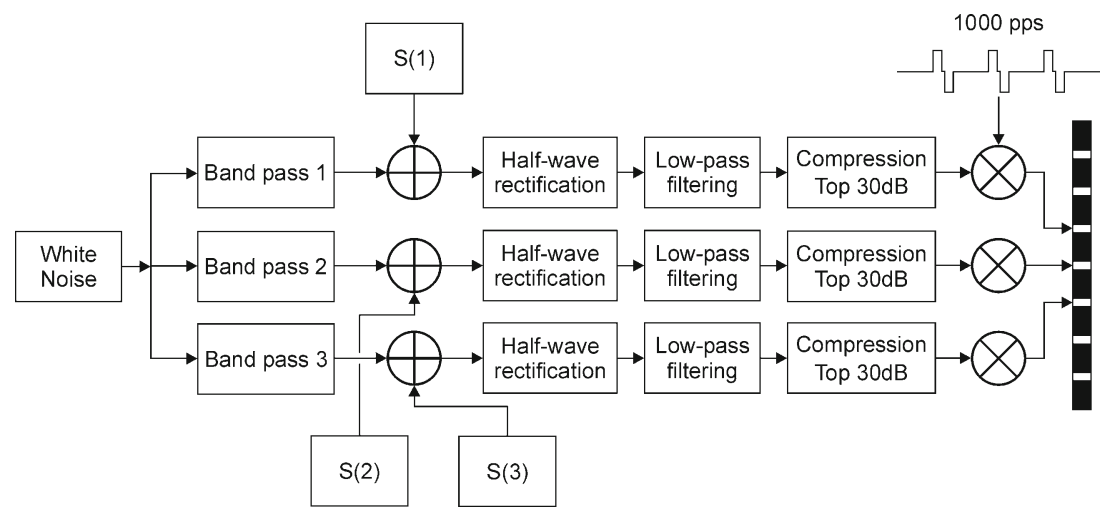

FIG. 1. Block diagram of the processing scheme for the BMLD stimuli, showing the conversion of digital signal $(S)$ and noise waveforms into electrical pulses. 

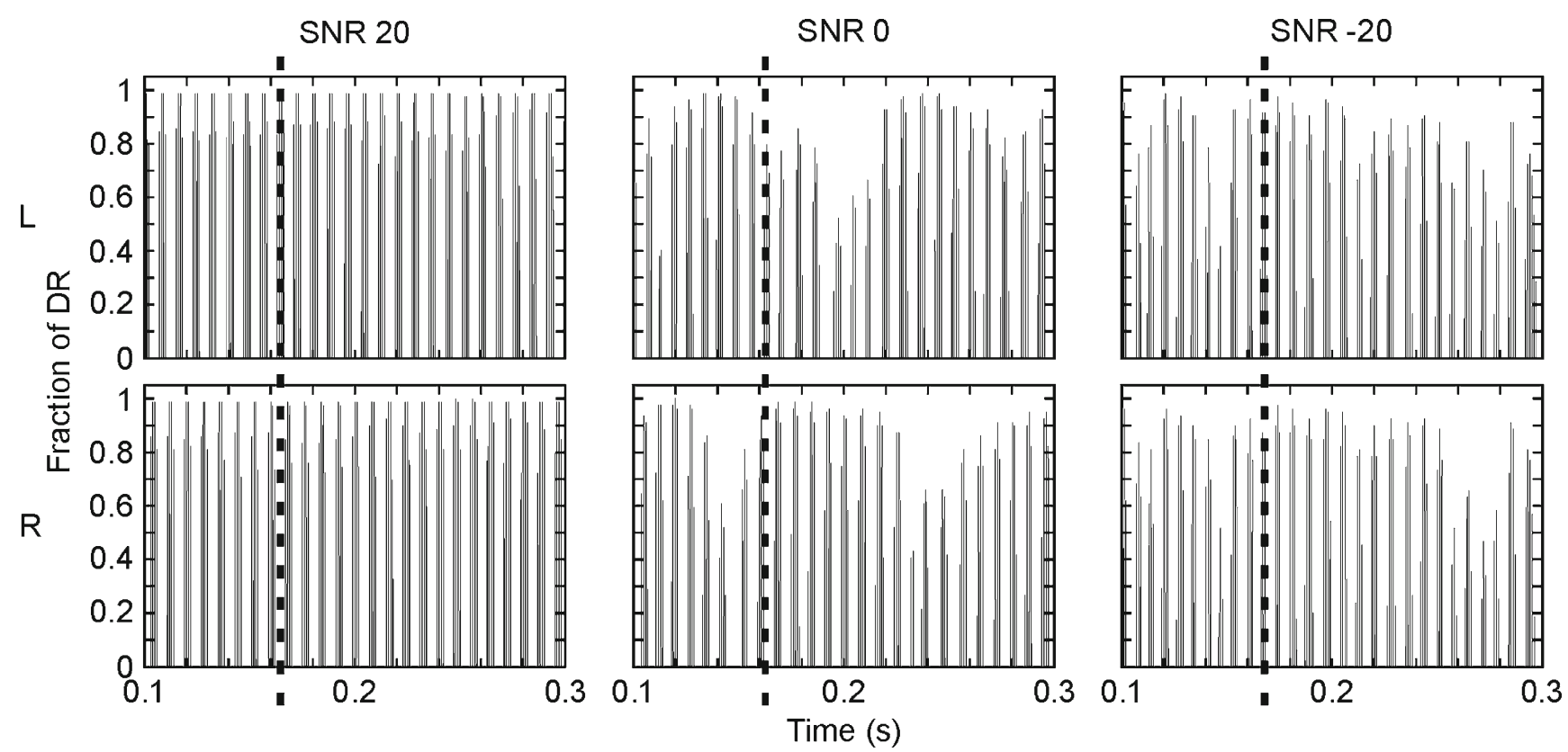

FIG. 2. Time-domain representation of the stimulus presented to the left $(L)$ and right $(R)$ electrodes in the $N_{0} S_{\tau}$ condition at different $S N R s$ (columns), after signal processing, including compression. The dashed line indicates a delay of $4 \mathrm{~ms}$ between the left and right ear. The pulse amplitude is expressed as a proportion of the dynamic range $(D R)$.

187.5 and $312.5 \mathrm{~Hz}$, and 312.5 and $437.5 \mathrm{~Hz}$ for channel 14, 13, and 12, respectively. The block diagram for multi-channel stimulation is shown in Figure 1. One white-noise token was band-pass filtered with fourth-order Butterworth filters to yield the three noise bands. A sinusoid was added to each noise band, after which the same processing as in the single-channel case was applied in each channel. A global maximum, determined across all three channels, was used for mapping the digitally sampled waveforms (top $30 \mathrm{~dB}$ ) into the electrical dynamic range. The three channels were stimulated in consecutive order, with a 71- $\mu$ s delay between individual pulses across channels. In order to prevent temporal shifting of the waveforms across channels as a result of the interleaved stimulation, the moment of sampling corresponded to the moment of stimulation-in contrast to what is done in the clinical systems, where channels are stimulated alternately, but sampled simultaneously. Interaural delays of 2 and $0.7 \mathrm{~ms}$ were used. In contrast to the $4-\mathrm{ms}$ delay, these values are not multiples of any of the periods of the harmonic signals and thus evoke differences between the signals in the left and right ear in each channel. The stimulation pattern for a 2-ms-delayed $\mathrm{N}_{0} \mathrm{~S}_{\tau}$ stimulus is shown in Figure 3, with the three rows representing the three channels and the columns representing different SNRs. The multichannel stimulus was chosen for its correspondence with speech signals. The stimulus is a multi-channel model for CI presentation of a vowel (three-harmonic complex) in a broadband $(375 \mathrm{~Hz})$ steady noise. The frequency of $125 \mathrm{~Hz}$ is in the range of fundamental frequencies of a male voice and the bandwidth of $125 \mathrm{~Hz}$ is equal to the bandwidth of the lower frequency channels in the Nucleus CI processors. Note that, in addition to the number of channels, the stimulation frequencies and the noise bandwidth are different from-and more realistic than-the single-channel situation.

No pitch matching was performed to determine electrodes that were matched in cochlear position across ears, because correspondence in pitch is not necessarily an indication of correspondence in cochlear position and of optimal binaural sensitivity (Long et al. 2003). Moreover, pilot tests had shown that BMLDs are not necessarily abolished by inaccurate place matching of electrodes (Van Deun 2009).

\section{Stimulation levels}

The following steps were carried out to select appropriate stimulation levels. First, detection thresholds were measured with an unmodulated 1,000-pps pulse train. Second, the most comfortable level for each ear was determined for the modulated pulse trains, processed in the same way as for the BMLD test, including compression, but without noise. Third, a loudness balancing task was performed with this stimulus. The level on the left side was kept fixed at the most comfortable level of step 2. For the right side, seven levels around the established comfort level were selected. In a constant-stimuli experiment, the 

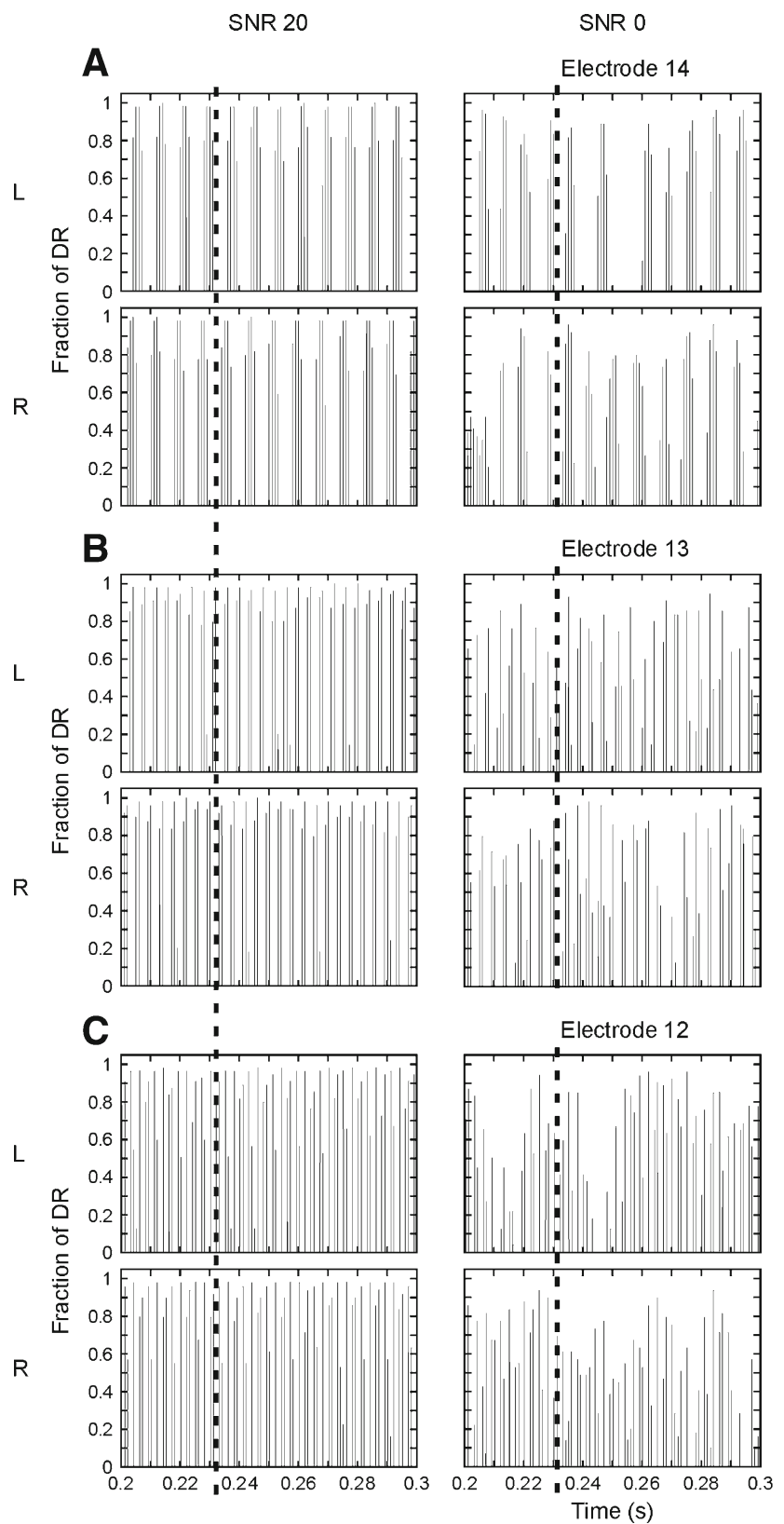

$\mathrm{L}$

L

$\mathrm{R}$
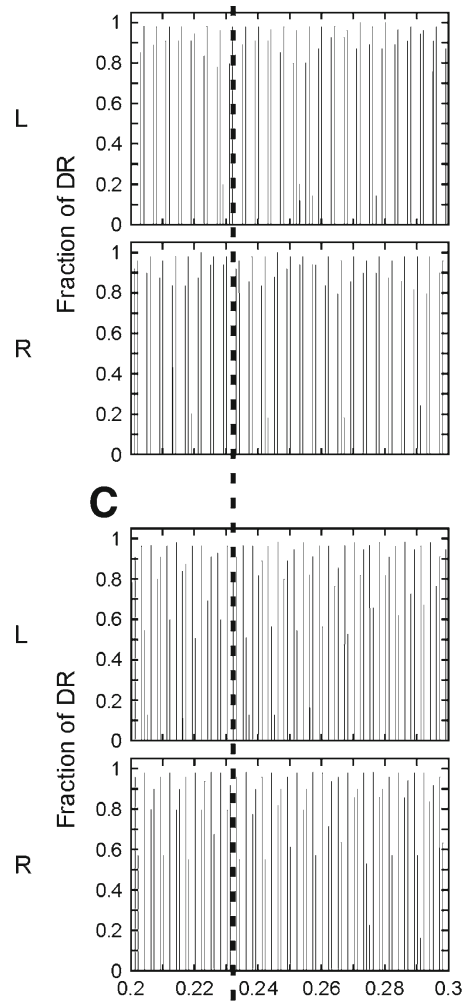

modulated stimulus was presented four times at each of the seven interaural level differences (with fixed level in the left ear), to both ears simultaneously. In the case of multi-channel stimulation, threshold and comfort levels were determined for all three electrodes together, and all three (adjacent) electrodes were stimulated with the same current levels. The listener had to indicate the side at which the stimulus was perceived (verbally or by pointing). To find the intensities leading to a centered percept (level for which 50\% "left" and 50\% "right" responses were given), a logistic psychometric function was fitted using the psignifit toolbox version 2.5.6 for Matlab, relating the proportion of "left" answers to the intensity in the right ear. The psignifit function implements the maximum-likelihood method described by Wichmann and Hill (2001a). Confidence intervals for
SNR -20

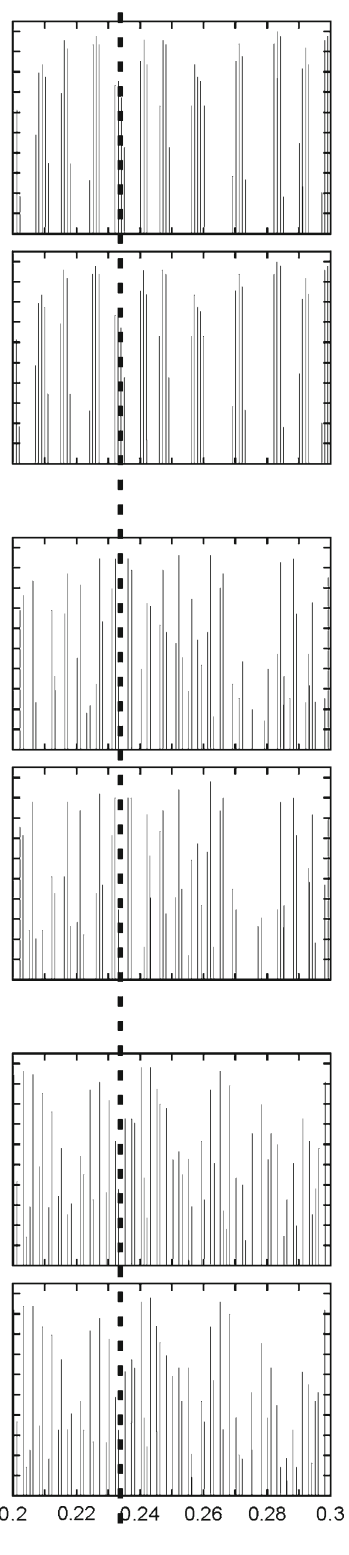

FIG. 3. Electrical stimulation patterns applied to the left $(L)$ and right $(R)$ ear at A electrode 14, B electrode 13, and C electrode 12, for the $\mathrm{N}_{0} \mathrm{~S}_{\tau}$ stimulus at different SNRs (columns). The dashed line indicates a delay of $2 \mathrm{~ms}$ between the left and right ear. The pulse amplitude is expressed as a proportion of the dynamic range $(D R)$. the $50 \%$ point were found by the BCa bootstrap method implemented by psignifit, based on 1999 simulations (Wichmann and Hill 2001b). Within 0.5-1 h, reliable threshold, comfortable and loudness-balanced levels could be determined for each stimulus type. Across subjects and stimulus types, the $95 \%$ confidence interval for the equal-loudness point had a mean width of three current units (range, 0-7 current units).

\section{BMLD measurement}

Thresholds were determined with a three-interval threealternative forced-choice task in an adaptive, two-down one-up procedure. Separate adaptive runs were conducted for diotic and dichotic conditions. The listener's task was to indicate which of three intervals with noise 
contained an additional signal (diotic or dichotic, depending on the condition). Noise tokens differed across intervals and trials. To familiarize subjects with this task, 10 trials in both the diotic and dichotic (4-ms delay) single-channel condition were administered at a fixed SNR of $20 \mathrm{~dB}$. The adaptive test started at an SNR of 20 (single-channel conditions) or $28 \mathrm{~dB}$ (multichannel conditions) and the SNR was adapted in steps of 8,4 , and $2 \mathrm{~dB}$ at the start, after two reversals, and after four reversals, respectively. A run was stopped after eight reversals. For each condition (diotic, 4-ms, 2-ms, or 0.7 -ms delay) and stimulus type (single/multi-channel), at least three runs were performed. Total testing time for the four single-channel (diotic, 4-ms, 2-ms, or 0.7-ms delay) and three multi-channel (diotic, 2-ms, or 0.7-ms delay) conditions together was about $8 \mathrm{~h}$ per subject. Diotic and dichotic runs for one stimulus type were administered in random order, whereas different stimulus types were assessed in separate test sessions. Note that the 4-ms single-channel condition was also included in our previous study on binaural unmasking in CI children (Van Deun et al. 2009). However, in that study thresholds were measured at fixed SNRs. Differences between previous and present results can be explained by differences in procedure (adaptive vs fixed) and by the time delay (about 2 years) between both studies. To be able to reliably compare data with the new 2-ms- and 0.7 -ms-delayed signals and the multi-channel conditions, new adaptive measurements for the 4-ms delay were included in the present study.

To determine threshold values, the performanceintensity function for each condition was tracked on the basis of the scores at the different presentation levels. Therefore, results of the different runs were pooled per condition and subject. Subsequently, a logistic function was fitted by the maximum-likelihood method of Wichmann and Hill (2001a). The threshold was determined as the SNR at $70 \%$ correct. Confidence intervals (90\%) were found by the BCa bootstrap method, based on 1,999 simulations (Wichmann and Hill 2001b). The adaptive method was used for determining data points at threshold level in an efficient way. The maximum-likelihood method allowed taking into account performance at other levels of difficulty and determining confidence intervals on our estimates.

\section{RESULTS}

Single- and multi-channel thresholds and BMLDs

The results for the single-channel conditions are shown in Figure 4. Thresholds at $70 \%$ correct for the diotic conditions are represented by yellow bars, those for the dichotic conditions by purple (4-ms delay), blue (2-ms delay), and green bars (0.7-ms delay). Error bars depict the $90 \%$ confidence inter-

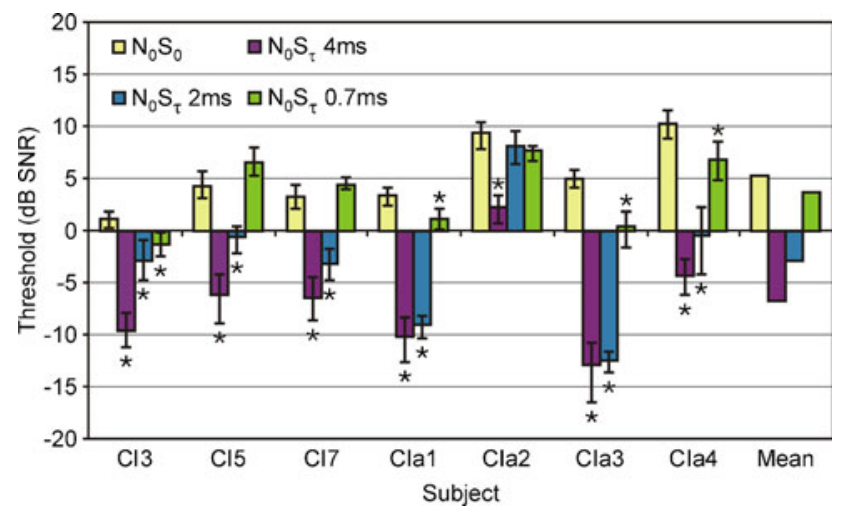

FIG. 4. Signal detection thresholds (in decibels SNR) for a singlechannel stimulus in diotic conditions $\left(\mathrm{N}_{0} \mathrm{~S}_{0}\right.$ - yellow) and conditions with a 4-ms $\left(\mathrm{N}_{0} \mathrm{~S}_{\tau} 4 \mathrm{~ms}\right.$ - purple), 2-ms $\left(\mathrm{N}_{0} \mathrm{~S}_{\tau} 2 \mathrm{~ms}\right.$-blue), and 0.7$\mathrm{ms}\left(\mathrm{N}_{0} \mathrm{~S}_{\tau} 0.7 \mathrm{~ms}\right.$ - green) delay. Error bars depict $90 \%$ confidence intervals. Non-overlapping error bars indicate significant differences (also indicated with stars).

vals. Non-overlapping intervals indicate significant differences between conditions $(p<0.05)$. Significant BMLDs (differences between dichotic and diotic conditions) are also indicated with a star.

It is clear from this figure that all subjects obtained a significant, positive BMLD for the 4-ms delay. The mean value was $12.0 \mathrm{~dB}$. The BMLD was smaller for the 2-ms delay, with a mean value of $8.1 \mathrm{~dB}$. Only for one subject (CIa2), the 2-ms BMLD was not significant. For a delay of $0.7 \mathrm{~ms}$, four out of seven subjects (CI3, CIa1, CIa3, and CIa4) had a significant BMLD of 2 to $5 \mathrm{~dB}$. Negative BMLDs were not significant in any of the subjects (overlapping error bars). Pairedsamples $t$ tests considering differences between diotic and dichotic conditions across all subjects indicated significant effects for the 4-ms delay $(t=8.88, p<0.001)$ and 2-ms delay $(t=3.84, p=0.009)$, but not for the 0.7 -ms delay $(t=1.70, p=0.140)$.

Figure 5 shows the results for the multi-channel conditions. Diotic thresholds are again represented by

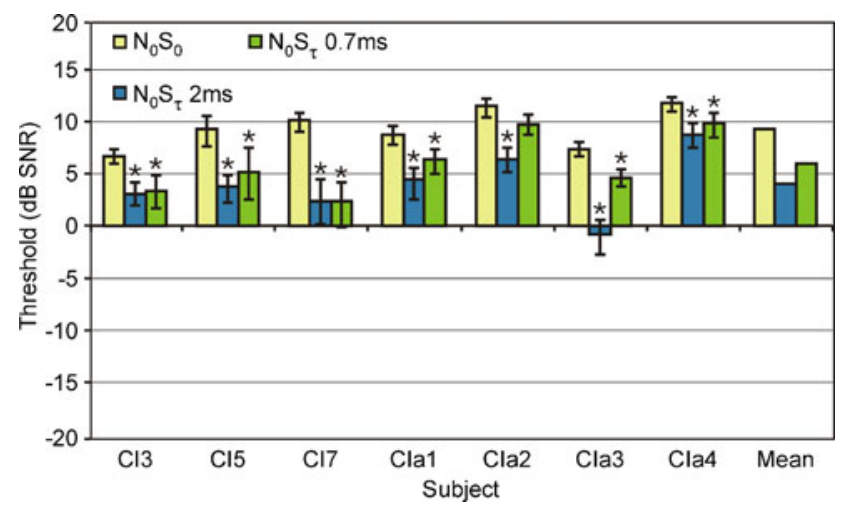

FIG. 5. Signal detection thresholds (in decibels SNR) for a multichannel stimulus in diotic conditions $\left(\mathrm{N}_{0} \mathrm{~S}_{0}-\right.$ yellow) and conditions with a 2-ms $\left(\mathrm{N}_{0} \mathrm{~S}_{\tau} 2 \mathrm{~ms}\right.$-blue) and 0.7-ms $\left(\mathrm{N}_{0} \mathrm{~S}_{\tau} 0.7 \mathrm{~ms}\right.$-green) delay. Error bars depict $90 \%$ confidence intervals. Non-overlapping error bars indicate significant differences (also indicated with stars). 
yellow bars; dichotic thresholds by blue ( $2 \mathrm{~ms})$ and green $(0.7 \mathrm{~ms})$ bars. Generally, detection thresholds were higher for the multi-channel than for the singlechannel conditions, with an average difference of $4.4 \mathrm{~dB}$. Significant BMLDs were found for all seven subjects for the 2-ms delay and for six subjects for the 0.7 -ms delay (not for CIa2), with average values of $5.4 \mathrm{~dB}(n=7)$ and $3.7 \mathrm{~dB}(n=6)$, respectively. Considered across the whole group, multi-channel BMLDs were significant for both the 2-ms delay $(t=7.219, p<$ $0.001)$ and the 0.7 -ms delay $(t=4.430, p=0.004)$.

A repeated-measures ANOVA with interaural condition (diotic, 2-ms delay, and 0.7-ms delay) and stimulus type $(125 \mathrm{~Hz}$ with $50-\mathrm{Hz}$-wide noise on one channel versus $125 / 250 / 375 \mathrm{~Hz}$ with 125 Hz-wide noise bands on three channels) as withinsubjects factors showed an overall effect of interaural delay $(F=21.32, p=0.004)$ and an overall effect of stimulus type $(F=23.98, p=0.003)$. The interaction was not significant $(F=3.53, p=0.110)$. Post hoc comparisons - with Bonferroni correction-for the three delay conditions revealed that the threshold was significantly smaller for the 2-ms-delayed compared to the diotic condition (significant BMLD of $6.8 \mathrm{~dB}, p=0.004)$ and for the 0.7 -ms-delayed compared to the diotic condition (significant BMLD of $2.5 \mathrm{~dB}, p=0.001)$. The difference between the 2-msand 0.7 -ms-delayed thresholds was also significant (difference $=4.3 \mathrm{~dB}, p=0.020$ ). When only the singlechannel conditions were considered, it appeared that the 4-ms-delayed stimulus was significantly better detected than the 2-ms- (difference $=3.8 \mathrm{~dB}$, $p=0.032$ ) and 0.7 -ms-delayed stimuli (difference $=$ $10.4 \mathrm{~dB}, p<0.001)$.

Figure 6 shows boxplots of the BMLDs (difference between diotic and dichotic condition) for single- and multi-channel conditions, for delays of 2 and $0.7 \mathrm{~ms}$.

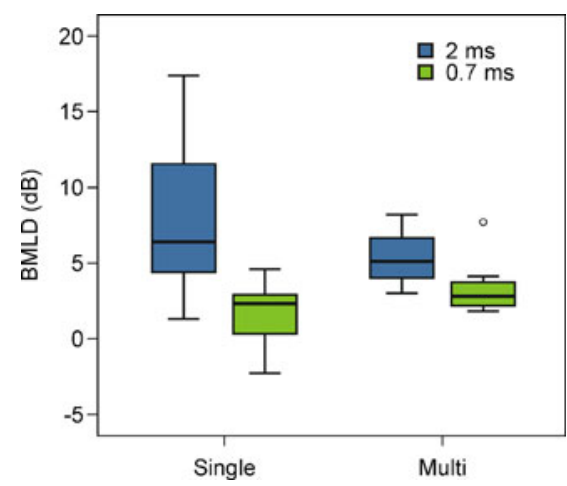

FIG. 6. Box plots of single- (left side) and multi-channel (right side) BMLDs for a 2-ms (blue) and 0.7-ms delay (green). Boxes represent the median (thick horizontal line), lower and upper quartiles (ends of boxes), minimum and maximum values (ends of whiskers), outliers (values between 1.5 and 3 times the interquartile range below the first quartile or above the third quartile-circles).
Although absolute thresholds were higher for the multi-channel compared to single-channel conditions, BMLDs were not significantly reduced (difference $=$ $0.5 \mathrm{~dB}, F=0.08, p=0.785)$.

\section{Exploring stimulus parameters}

Different stimulus parameters were changed in the multi- compared to single-channel experiment. To determine the effect of individual parameters and to find out which parameters affected performance and which did not, additional stimulus conditions were explored in four subjects (CI7, CIa1, CIa2, and CIa3). Each time only one stimulus parameter was varied compared to the standard single-channel situation of a $125-\mathrm{Hz}$ sinusoid with a $4-\mathrm{ms}$ delay in a 50-Hz-wide noise band.

First, the effect of inducing faster envelope fluctuations was investigated by raising the frequency of the sinusoid from 125 to $375 \mathrm{~Hz}$. The $375-\mathrm{Hz}$ stimulus was presented to a single channel in each ear with a delay of $2 \mathrm{~ms}$, in a noise band of $50 \mathrm{~Hz}$ wide (cutoff frequencies of 350 and $400 \mathrm{~Hz}$ ). Results of four subjects (CI7, CIa1, CIa2, and CIa3) are shown in Figure 7A. The $375-\mathrm{Hz}$ sinusoid evoked similar thresholds and BMLDs (all significant) to the $125-\mathrm{Hz}$ sinusoid, with a mean value of $10.3 \mathrm{~dB}\left(\mathrm{~N}_{0} \mathrm{~S}_{\tau}\right.$ threshold: $t=-0.82, p=0.473$; BMLD: $t=1.32, p=0.280)$.

In a second step, the bandwidth of the noise was increased, to better correspond with natural/clinical values. An increase in bandwidth has the effect of raising the frequency and reducing the depth of the envelope fluctuations. The same four subjects performed the single-channel BMLD test with a $125-\mathrm{Hz}$ signal in a $375-\mathrm{Hz}$-wide noise (center frequency of $250 \mathrm{~Hz}$, cutoff frequencies of 62.5 and $437.5 \mathrm{~Hz}$ ), with an interaural delay of $4 \mathrm{~ms}$ (Figure 7B). An average BMLD of $9.0 \mathrm{~dB}$ was found (significant for each subject), which was not significantly different from that for the $50-\mathrm{Hz}$-wide noise $\left(\mathrm{N}_{0} \mathrm{~S}_{\tau}\right.$ threshold: $t=0.95, p=0.413$; BMLD: $t=-1.23, p=0.307$ ).

Third, the effect of adding pulses to adjacent implant electrodes while keeping the signal information constant across channels was measured by stimulating three channels with the same $125-\mathrm{Hz}$ sinusoid in the 50-Hz-wide noise band ("uniform" stimulus). A delay of $2 \mathrm{~ms}$ was used to be able to compare data with those for the single-channel stimulus as well as those for the multi-channel harmonic complex. Three subjects (CIa1, CIa2, and CIa3) performed the BMLD task in this condition. In Figure 7C, results are compared with those from the single-channel case. Thresholds and BMLDs were similar for single- and multi-channel stimulation $\left(\mathrm{N}_{0} \mathrm{~S}_{\tau}\right.$ threshold: $t=0.63, p=0.594$; 


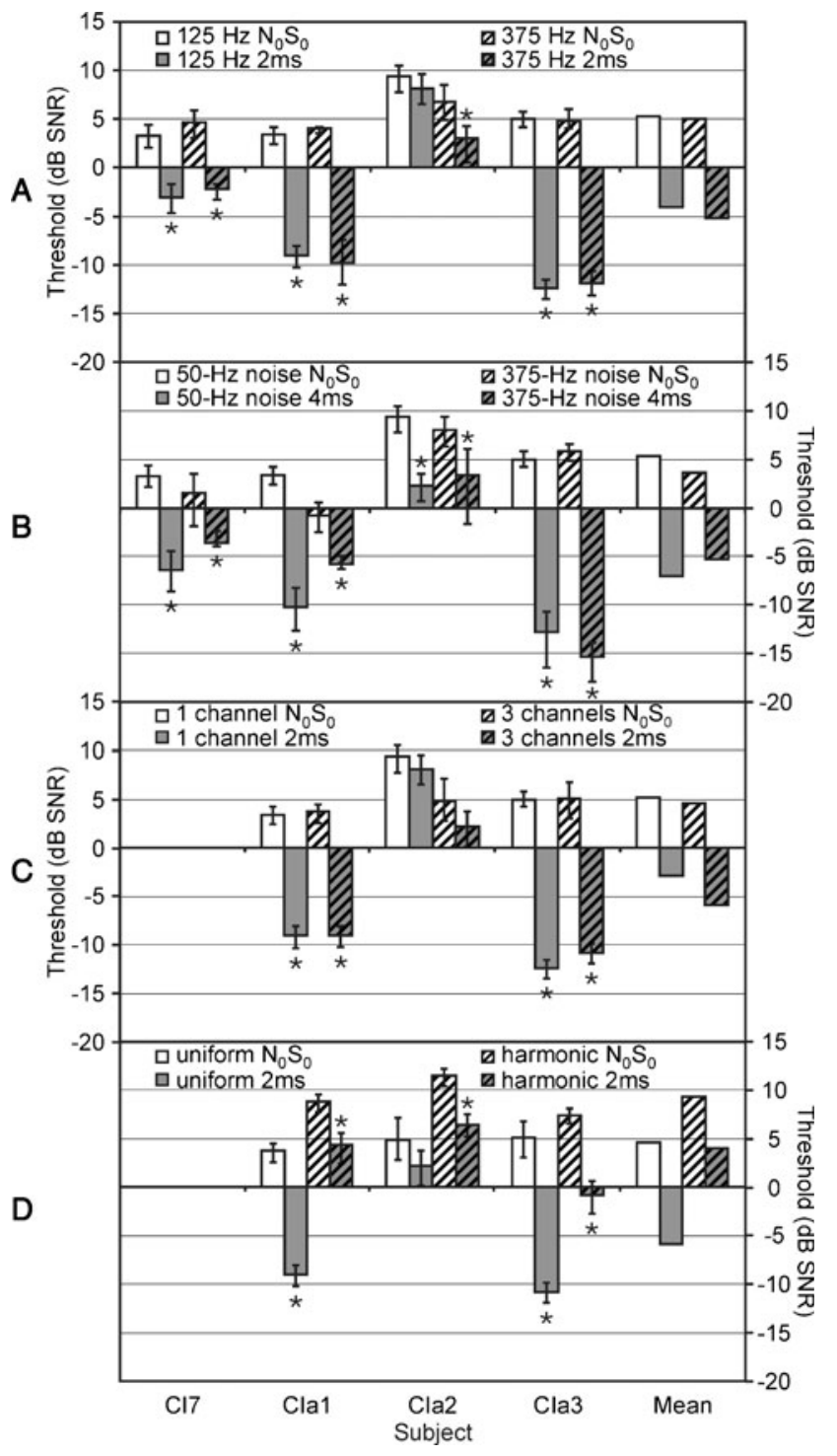

FIG. 7. Signal detection thresholds (in decibels SNR) in diotic $\left(\mathrm{N}_{0} \mathrm{~S}_{0}\right.$-white) and dichotic $\left(\mathrm{N}_{0} \mathrm{~S}_{\tau}\right.$-gray) conditions for $\mathbf{A}$ different sine wave frequencies $(125 \mathrm{~Hz}$-plain bars versus $375 \mathrm{~Hz}$-hatched bars), B different noise bandwidths ( $50 \mathrm{~Hz}$-plain bars versus $375 \mathrm{~Hz}$ - hatched bars), C different number of channels (one-plain bars versus three-hatched bars), and D uniform stimulus (plain bars) versus harmonic complex (hatched bars) on three channels. The size of the interaural delay in the $\mathrm{N}_{0} \mathrm{~S}_{\tau}$ condition was dependent on the stimulus type and is indicated in the legend. Error bars depict $90 \%$ confidence intervals. Non-overlapping error bars indicate significant differences (also indicated with stars).

BMLD: $t=-0.12, p=0.971)$. BMLDs were significant for two of three subjects (CIa1 and CIa3). When the multi-channel harmonic complex was compared with the uniform multi-channel stimulus (Figure 7D), the BMLD was reduced by $4.6 \mathrm{~dB}$ but was significant in all three subjects. The difference with uniform stimulation was not significant in this small group $\left(\mathrm{N}_{0} \mathrm{~S}_{\tau}\right.$ threshold: $t=-3.43$, $p=0.076$; BMLD: $t=1.31, p=0.321)$.

\section{DISCUSSION}

In this study, binaural unmasking was established for single- and multi-channel electrical stimulation with varying interaural delay. The binaural benefit decreased with decreasing interaural delay but was still present for the harmonic complex with a 700$\mu$ s delay. These results show that binaural sensitivity can be evoked with controlled electrical stimulation.

\section{Binaural cues}

It is unsure whether listeners were using the time delay on itself to obtain better detection thresholds in the dichotic conditions, or whether they relied on other cues that resulted from this delay. There are reasons to believe that the contribution of level cues was very small and that they do not explain the observed benefits. Monaural level cues were reduced by the use of different noise tokens in each stimulation interval. Moreover, they were similar for diotic and dichotic stimulation and therefore cannot explain the BMLD. Level differences between the ears, to the contrary, were present in the dichotic stimuli but not in the diotic ones. However, based on the following arguments we can state that the BMLD was probably not based on these interaural level differences (ILDs). On the one hand, instantaneous ILDs are expected to be eliminated by slow temporal integration. Long-time averaged ILDs, on the other hand, are very small and do not comprise a consistent cue. This can be shown by estimating the detection thresholds based on a model which contains the actual interaural level differences present in the stimuli (expressed as electrical levels in decibels, after filtering and compression) and the just noticeable difference in ILD for electrical stimulation. Literature reports that ILDs as small as one to four current units or 0.09 to $0.69 \mathrm{~dB}$ can be detected by some listeners (Lawson et al. 1998; van Hoesel and Tyler 2003). Average ILDs in our dichotic stimuli comprised $0.1 \mathrm{~dB}$ - with a standard deviation of $0.1 \mathrm{~dB}$ - for an average dynamic range of 65 current units. This means that the ILD may have been perceivable in some stimuli, but it was not a consistent cue. To demonstrate this, a Monte Carlo simulation of the adaptive 3-AFC procedure was performed. The simulation contained a detection algorithm based on ILD, in which the correct interval was selected for ILDs larger than $0.09 \mathrm{~dB}$, and a random interval (chance of correct response of $1 / 3$ ) was selected for smaller ILDs. The average detection threshold for the dichotic conditions based on this simulation (1,000 repetitions) was $18.0 \mathrm{~dB}$, with a standard deviation of $1.8 \mathrm{~dB}$. This threshold is significantly higher than the observed 
thresholds in our experiments, such that it is very unlikely that the BMLD was based on ILDs.

\section{Factors affecting sensitivity}

Multi-channel stimulation was performed with higher frequencies and larger noise bandwidths compared to our previous single-channel experiments (Van Deun et al. 2009). It was assumed that the faster and less pronounced envelope fluctuations associated with the extended frequency range would reduce the salience of interaural envelope cues and lead to smaller BMLDs. Moreover, the channel interactions that occur when multiple, adjacent electrodes are stimulated were expected to distort the electrical stimulation pattern and envelope fluctuations within a channel and thereby reduce sensitivity to timing differences.

In our study, BMLDs were not significantly smaller for the multi-channel stimuli. However, for some subjects BMLDs were reduced and for all of them overall thresholds were elevated compared to the single-channel case. As mentioned before, several parameters were changed when going from singleto multi-channel stimulation. To find out which of them affected performance and which did not, additional stimulus conditions were administered to a subgroup of the subjects.

These experiments showed that faster envelope fluctuations (related to higher sinusoid frequencies or broader noise bands) do not necessarily harm binaural sensitivity. It cannot be excluded that thresholds might start to degrade for higher frequencies and larger bandwidths than the ones employed here. For the case of acoustic BMLDs in normal-hearing listeners, varying dependencies on frequency have been shown, but generally BMLDs are large up to $500 \mathrm{~Hz}$ and start to degrade thereafter (Hall and Harvey 1985; Hirsh 1948; Kohlrausch 1986). In a recent study of van Hoesel et al. (2009) with electrical stimulation, a modulation rate of $300 \mathrm{~Hz}$ resulted in poorer ITD-based lateralization than a rate of $100 \mathrm{~Hz}$. The discrepancy with our results might be related to individual differences, given the high variability that is observed for these tasks. Note that some subjects in the study of van Hoesel et al. obtained similar performance for 100 and $300 \mathrm{~Hz}$. Also differences in the applied perceptual tasks may play a role. A certain interaural delay might be perceived as a decorrelation between the signals in both ears although the precise direction of the shift (lateralization task) is not perceivable. In the BMLD task, it was not necessary to lateralize the sounds.

Although the difference in BMLD between the harmonic complex and uniform multi-channel stimuli was not significant, the introduction of different stimulation patterns in different channels seemed to evoke the largest effect on thresholds and BMLDs.
The reason for this is unknown, but it is conceivable that spread of excitation and channel interaction play an important role here. Responses to pulses delivered to adjacent electrodes may distort the temporal pattern within a channel and reduce envelope fluctuations. Literature is inconclusive about the overlap between neighboring channels in current CIs. McKay and McDermott (1996) showed that when two electrodes within 3 to $4 \mathrm{~mm}$ are stimulated alternately, the aggregate temporal pattern is perceived by the CI user instead of the individual patterns. On the other hand, in a more recent study, Macherey and Carlyon found no aggregate perception of pitch for spacings as small as $0.75 \mathrm{~mm}$ (Macherey and Carlyon 2010). Electrodes in the Nucleus implants that were involved in the current study are spaced $0.75 \mathrm{~mm}$ apart. Spread of excitation may have affected excitation patterns in some or all subjects, although no definite conclusions can be drawn from the data. Even with the same stimulation pattern on all channels, spread of excitation might lead to a poorer representation of the stimulus fluctuations by increasing the effective stimulation rate at each electrode and thereby creating elevated refractory effects. In our setup, with a stimulation rate of $1,000 \mathrm{pps}$ in each channel and a total rate of $14,000 \mathrm{pps}$, no such effect was observed, since multichannel thresholds and BMLDs for the uniform stimulus were similar to single-channel thresholds and BMLDs. Binaural sensitivity was reduced only with differential stimulation across channels.

It could be argued that elevated thresholds and reduced BMLDs with multi-channel stimulation were caused by modulation detection interference (MDI). MDI refers to the elevation of modulation detection/ discrimination thresholds by the presence of a second modulated signal, which is not limited to critical-band or auditory-filter processing but is assumed to comprise more central interactions (Yost and Sheft 1989). MDI has also been demonstrated in cochlear implant users (Chatterjee 2003; Richardson et al. 1998). It has been shown that MDI is unaffected by or decreases with the distance between frequencies (Mendoza et al. 1995; Yost and Sheft 1989). In our study, MDI did not appear to contribute significantly. If that was the case, we would have observed elevated thresholds and reduced BMLDs also (and especially) for the uniform stimulus.

An interesting finding is that for the 700- $\mu$ s delay, more subjects had a significant BMLD when multiple channels were stimulated (four significant results) than for single-channel stimulation (six significant results). This can possibly be explained by the fact that, for the higher frequencies included in the multichannel condition, the $700-\mu$ s delay constitutes a larger proportion of the signal period and leads to a larger decorrelation between ears. In other words, the 
same time delay corresponds to a larger phase delay for higher frequencies. For the $125-\mathrm{Hz}$ single-channel stimulus, the 2-ms delay resulted in a large interaural decorrelation whereas only subtle interaural differences were created by the 700- $\mu$ s delay. An additional measurement was performed to test the hypothesis that the better multi- than single-channel results for $700 \mu$ s were attributable to the higher frequency components yielding larger BMLDs. A single-channel $375-\mathrm{Hz}$ stimulus was presented with a delay of $700 \mu$ s to subject CI7. A large, significant BMLD of $10.9 \mathrm{~dB}$ was found, confirming our hypothesis. The same process can explain the similar BMLD for the $375-\mathrm{Hz}$ and the $125-\mathrm{Hz}$ single-channel stimulus with a delay of $2 \mathrm{~ms}$. This delay covers respectively $3 / 4$ and $1 / 4$ of the signal periods. These proportions lead to a similar amount of interaural decorrelation, albeit with a different ear leading.

\section{Individual variability}

Similar to other research on CI outcomes, a rather high variability was observed among individual CI users. Part of this variability may be attributable to a better or worse selection of interaural electrode pairs, since no individualized matching was performed. However, similar variability has been observed in previous studies where electrodes had been matched interaurally (Long et al. 2006; Lu et al. 2010). Interestingly, multi-channel BMLDs were obtained both by adults with postlingual onset of deafness and by children with prelingual onset of deafness. Two of the children in this study had some access to acoustic cues in the early years of life, either because of later onset of deafness as a result of meningitis (CI5, 9 months) or because of progressive hearing loss (CI7). Subject CI3, however, was born deaf as a result of congenital CMV infection, and never had access to acoustic stimulation. Early implantation reduced degeneration of the neural system and allows the child to benefit from binaural electrical stimulation. Recent studies indicate that outcomes might be different without stimulation in the early years of life (Litovsky et al. 2010; Poon et al. 2009).

\section{Implications and future research}

As was demonstrated in this study, interaural cues in the signal envelope can contribute significantly to binaural sensitivity through bilateral electrical stimulation. Findings are promising in view of real-life benefits. Although it is expected that higher frequencies $(>1 \mathrm{kHz})$ and larger bandwidths will result in less binaural unmasking because of the incapability of the central auditory system to follow faster envelope fluctuations (Bernstein and Trahiotis 1994), information from the lowest channels may be sufficient to obtain benefits for signal detection in noise. Stimuli in this study were processed in a manner similar to current speech processing strategies, except for the specific implementation of rectification and the coordination of stimulation across ears. The present results suggest that coordinating the stimulation between clinical speech processors, together with emphasizing envelope fluctuations, may yield important benefits for signal detection. The importance of coordinating individual pulses cannot directly be derived from this study, since a relatively high carrier pulse rate was used and no timing information was encoded. However, the coordination of processors ensured that no general, device-dependent processing delays were introduced and that the timing of envelopes was coordinated at both ears. In addition to the coordination of stimulation, the accentuation of envelope cues resulting from the half-wave rectification-comparable to the transposed stimuli as used by van de Par and Kohlrausch (1997) and Bernstein and Trahiotis (2002) - might have been an important facilitator of binaural sensitivity. This factor might partly explain why BMLDs were found whereas previous studies did not show BILDs with coordinated stimulation. Further research is needed, however, to directly assess the importance of the coordinated stimulation and half-wave rectification. Another difference with previous studies is the nature of the task and stimuli. It remains to be investigated whether the effect that was established here also holds for speech perception in noise. From normal-hearing literature, it is known that binaural unmasking is generally smaller in understanding (binaural intelligibility level difference) compared to detection tasks (binaural masking level difference) (Blauert 1997). This can partly be explained by the dominant frequency region of speech, which is situated in the mid-to-high frequencies, whereas BMLDs are highest for low-frequency signals, around $500 \mathrm{~Hz}$. Moreover, as already mentioned before, detection and understanding are different tasks which may tap into different auditory and cognitive processes. Nevertheless, a small benefit can mean a significant improvement for the perception but also the detection of speech in noise. Future studies could implement the applied processing scheme-with enhanced envelope fluctuations resulting from the half-wave rectification - in experimental binaural speech processors to evaluate speech perception under direct stimulation and in free field.

\section{CONCLUSIONS}

This paper investigated sensitivity to multi-channel interaural cues in prelingually and postlingually 
deafened bilateral cochlear implant users. Binaural unmasking was observed for stimulation of multiple adjacent channels with realistic interaural delays. These findings are promising in view of real-life benefits. It remains to be demonstrated whether the benefits are also observed for speech perception, under realistic circumstances, and to which extent coordination of bilateral speech processors and the enhancement of envelope fluctuations contribute to these results.

\section{ACKNOWLEDGMENTS}

The authors would like to thank the team of the Medical University of Hannover for access to the patients and for their help. We thank all patients for their time and efforts. This work was supported by postdoctoral mandates (BOF) from the Research Council of the K.U.Leuven to the first and third author and by IWT Vlaanderen (project 080304).

\section{Conflict of interest disclosure statement}

The authors declare that there are no conflicts of interest related to the work presented in the submitted paper "Binaural unmasking of multi-channel stimuli in bilateral cochlear implant users".

\section{REFERENCES}

Bernstein LR, Trahiotis C (1994) Detection of interaural delay in highfrequency sinusoidally amplitude-modulated tones, two-tone complexes, and bands of noise. J Acoust Soc Am 95:3561-3567

Bernstein LR, Trahiotis C (2002) Enhancing sensitivity to interaural delays at high frequencies by using "transposed stimuli". J Acoust Soc Am 112:1026-1036

Blauert J (1997) Spatial hearing. The psychophysics of human sound localization. MIT, Massachusetts

Buss E, Pillsbury HC, Buchman CA, Pillsbury CH, Clark MS, Haynes DS, Labadie RF, Amberg S, Roland PS, Kruger P, Novak MA, Wirth JA, Black JM, Peters R, Lake J, Wackym PA, Firszt JB, Wilson BS, Lawson DT, Schatzer R, D'Haese PS, Barco AL (2008) Multicenter U.S. bilateral MED-EL cochlear implantation study: speech perception over the first year of use. Ear Hear 29:20-32

Chatterjee M (2003) Modulation masking in cochlear implant listeners: envelope versus tonotopic components. J Acoust Soc Am 113:2042-2053

Declaration of Helsinki. Adopted by the 18th World Medical Assembly, Helsinki, Finland, 1964 and revised by the 29th World Medical Assembly, Tokyo, Japan in 1975

Francart T, van Wieringen A, Wouters J (2008) APEX 3: a multipurpose test platform for auditory psychophysical experiments. J Neurosci Methods 172:283-293

Hall JW, Harvey AD (1985) The binaural masking level difference as a function of frequency, masker level and masking bandwidth in normal-hearing and hearing-impaired listeners. Audiology $24: 25-31$

HIRSH IJ (1948) The influence of interaural phase on interaural summation and inhibition. J Acoust Soc Am 20:536-544
Kohlrausch A (1986) The influence of signal duration, signal frequency and masker duration on binaural masking level differences. Hear Res 23:267-273

Laszig R, Aschendorff A, Stecker M, Müller-Deile J, Maune S, Dillier N, Weber B, Hey M, Begall K, Lenarz T, Battmer RD, Bohm M, Steffens T, Strutz J, Linder T, Probst R, Allum J, Westhofen M, Doering W (2004) Benefits of bilateral electrical stimulation with the nucleus cochlear implant in adults: 6-month postoperative results. Otol Neurotol 25:958-968

Lawson DT, Wilson BS, Zerbi M, Van den Honert C, Finley CC, FARmer JC JR, McElveen JT JR, Roush PA (1998) Bilateral cochlear implants controlled by a single speech processor. Am J Otol 19:758-761

Litovsky R, Parkinson A, Arcaroli J, Sammeth C (2006) Simultaneous bilateral cochlear implantation in adults: a multicenter clinical study. Ear Hear 27:714-731

Litovsky RY, Jones GL, Agrawal S, van Hoesel R (2010) Effect of age at onset of deafness on binaural sensitivity in electric hearing in humans. J Acoust Soc Am 127:400-414

Long CJ, Eddington DK, Colburn HS, Rabinowitz WM (2003) Binaural sensitivity as a function of interaural electrode position with a bilateral cochlear implant user. J Acoust Soc Am 114:1565-1574

Long CJ, Carlyon RP, Litovsky RY, Downs DH (2006) Binaural unmasking with bilateral cochlear implants. J Assoc Res Otolaryngol 7:352-360

Lu T, Litovsky R, Zeng FG (2010) Binaural masking level differences in actual and simulated bilateral cochlear implant listeners. J Acoust Soc Am 127:1479-1490

Macherey O, Carlyon RP (2010) Temporal pitch percepts elicited by dual-channel stimulation of a cochlear implant. J Acoust Soc Am 127:339-349

McKay CM, McDermott HJ (1996) The perception of temporal patterns for electrical stimulation presented at one or two intracochlear sites. J Acoust Soc Am 100:1081-1092

Mendoza L, Hall JW III, Grose JH (1995) Within- and acrosschannel processes in modulation detection interference. J Acoust Soc Am 97:3072-3079

Poon BB, Eddington DK, Noel V, Colburn HS (2009) Sensitivity to interaural time difference with bilateral cochlear implants: development over time and effect of interaural electrode spacing. J Acoust Soc Am 126:806-815

Richardson LM, Busby PA, Clark GM (1998) Modulation detection interference in cochlear implant subjects. J Acoust Soc Am 104:442-452

Schleich P, Nopp P, D'Haese P (2004) Head shadow, squelch, and summation effects in bilateral users of the MED-EL COMBI 40/ 40+cochlear implant. Ear Hear 25:197-204

VAn de Par S, Kohlrausch A (1997) A new approach to comparing binaural masking level differences at low and high frequencies. J Acoust Soc Am 101:1671-1680

Van Deun, L (2009) Binaural and spatial hearing in children with bilateral cochlear implants. Dissertation, K.U. Leuven, Belgium.

Van Deun L, van Wieringen A, Francart T, Scherf F, Dhooge IJ, Deggouj N, Desloovere C, Van de Heyning PH, Offeciers FE, De Raeve L, Wouters J (2009) Bilateral cochlear implants in children: binaural unmasking. Audiol Neurotol 14:240-247

Van Deun L, van Wieringen A, Scherf F, Deggouj N, Desloovere C, Offeciers FE, Van de Heyning PH, Dhooge IJ, Wouters J (2010A) Earlier intervention leads to better sound localization in children with bilateral cochlear implants. Audiol Neurotol 15:7-17

Van Deun L, van Wieringen A, Wouters J (2010B) Spatial speech perception benefits in young children with normal hearing and cochlear implants. Ear Hear 31:702-713 
van Hoesel RJ, Tyler RS (2003) Speech perception, localization, and lateralization with bilateral cochlear implants. J Acoust Soc Am 113:1617-1630

van Hoesel R, Bohm M, Pesch J, Vandali A, Battmer RD, Lenarz T (2008) Binaural speech unmasking and localization in noise with bilateral cochlear implants using envelope and fine-timing based strategies. J Acoust Soc Am 123:2249-2263

van Hoesel RJ, Jones GL, Litovsky RY (2009) Interaural time-delay sensitivity in bilateral cochlear implant users: effects of pulse rate, modulation rate, and place of stimulation. J Assoc Res Otolaryngol 10:557-567
Wichmann FA, Hill NJ (2001A) The psychometric function: I. Fitting, sampling, and goodness of fit. Percept Psychophys 63:1293-1313

Wichmann FA, Hill NJ (2001в) The psychometric function: II. Bootstrap-based confidence intervals and sampling. Percept Psychophys 63:1314-1329

Wilson BS, Finley CC, Lawson DT, Wolford RD, Eddington DK, RABINOWITZ WM (1991) Better speech recognition with cochlear implants. Nature 352:236-238

Yost WA, SHEFT S (1989) Across-critical-band processing of amplitudemodulated tones. J Acoust Soc Am 85:848-857 